\title{
Aneurysm Study of Pipeline in an Observational Registry (ASPIRe)
}

\author{
David F. Kallmes ${ }^{1} \quad$ Waleed Brinjikji ${ }^{1} \quad$ Edoardo Boccardi ${ }^{2}$ Elisa Ciceri ${ }^{3}$ \\ Orlando Diaz ${ }^{4}$ Rabih Tawk ${ }^{5}$ Henry Woo $^{7}$ Pascal Jabbour $^{9}$ \\ Felipe Albuquerque ${ }^{11}$ Rene Chapot ${ }^{13}$ Alain Bonafe ${ }^{15}$ Shervin R. Dashti ${ }^{16}$ \\ Josser E. Delgado Almandoz ${ }^{17}$ Curtis Given II $^{18} \quad$ Michael E. Kelly ${ }^{19}$ \\ DeWitte T. Cross III ${ }^{21}$ Gary Duckwiler ${ }^{22}$ Nasser Razack ${ }^{23}$ Ciaran J. Powers ${ }^{24}$ \\ Sebastian Fischer ${ }^{14}$ Demetrius Lopes ${ }^{25}$ Mark R. Harrigan 26 \\ Daniel Huddle ${ }^{27}$ Raymond Turner IV ${ }^{28}$ Osama O. Zaidat ${ }^{29}$ Luc Defreyne ${ }^{30}$ \\ Vitor Mendes Pereira ${ }^{20}$ Saruhan Cekirge ${ }^{31}$ David Fiorella ${ }^{8}$ Ricardo A. Hanel ${ }^{6}$ \\ Pedro Lylyk $^{32}$ Cameron McDougall $^{12}$ Adnan Siddiqui $^{10}$ Istvan Szikora ${ }^{33}$ \\ Elad Levy ${ }^{10}$
}

\footnotetext{
${ }^{1}$ Department of Radiology, Mayo Clinic, Rochester, Minn., USA; ${ }^{2}$ Department of Neuroradiology, Niguarda Ca' Granda Hospital of Milan, and ${ }^{3}$ Department of Radiology, Istituto Neurologico Carlo Besta, Milan, Italy; ${ }^{4}$ Department of Radiology, Houston Methodist Hospital, Houston, Tex., ${ }^{5}$ Department of Neurosurgery, Mayo Clinic, and ${ }^{6}$ Stroke and Cerebrovascular Surgery, Lyerly Neurosurgery/Baptist Neurological Institute, Jacksonville, Fla., Departments of Neurosurgery at ${ }^{7}$ Stony Brook University and ${ }^{8}$ Cerebrovascular Center, Stony Brook University Medical Center, Stony Brook, N.Y., ${ }^{9}$ Department of Neurosurgery, Thomas Jefferson University, Philadelphia, Pa., ${ }^{10}$ Department of Neurosurgery, University at Buffalo Neurosurgery, Buffalo, N.Y., and Departments of ${ }^{11}$ Neurosurgery and ${ }^{12}$ Endovascular Neurosurgery, Barrow Neurological Institute, Phoenix, Ariz., USA; ${ }^{13}$ Neurointerventional Services, Department of Interventional Neuroradiology, Alfried Krupp Hospital, Essen, and ${ }^{14}$ Department of Radiology, Klinikum Stuttgart, Stuttgart, Germany; ${ }^{15}$ Department of Radiology, CHU Montpellier, Montpellier, France; ${ }^{16}$ Department of Neurosurgery, Norton Neuroscience Institute, Norton Healthcare, Louisville, Ky., ${ }^{17}$ Department of Radiology, Neuroscience Institute, Abbott Northwestern Hospital, Minneapolis, Minn., and ${ }^{18}$ Neurointerventional Services, Baptist Health Lexington, Lexington, Ky., USA; ${ }^{19}$ Division of Neurosurgery, Royal University Hospital, University of Saskatchewan, Saskatoon, Sask., and ${ }^{20}$ Division of Neuroradiology, Joint Department of Medical Imaging and Division of Neurosurgery, Department of Surgery, University Health Network and Departments of Medical Imaging and Surgery, University of Toronto, Toronto, Ont., Canada; ${ }^{21}$ Department of Radiology, Washington University School of Medicine, St. Louis, Mo., ${ }^{22}$ Department of Neuroradiology, David Geffen School of Medicine at UCLA, Los Angeles, Calif., ${ }^{23}$ Neurointerventional Associates, P.A., St. Petersburg, Fla., ${ }^{24}$ Department of Neurological Surgery, The Ohio State University Wexner Medical Center, Columbus, Ohio, ${ }^{25}$ Department of Neurological Surgery, Rush University Medical Center, Chicago, Ill., ${ }^{26}$ Department of Neurosurgery, University of Alabama, Birmingham, Ala., ${ }^{27}$ Swedish Medical Center/RIA Neurovascular, Englewood, Colo., ${ }^{28}$ Department of Neurosurgery, Medical University of South Carolina, Charleston, S.C., and ${ }^{29}$ Department of Neurology, Medical College of Wisconsin/Froedtert Hospital, Milwaukee, Wis., USA; ${ }^{30}$ Department of Interventional Radiology, Ghent University Hospital, Gent, Belgium; ${ }^{31}$ Department of Radiology, Koru Hospital and Bayindir Hospitals, Ankara, Turkey; ${ }^{32}$ Department of Neurosurgery, Clinica La Sagrada Familia, ENERI, Buenos Aires, Argentina; ${ }^{33}$ Department of Neurointerventional Services, National Institute of Clinical Neurosciences, Budapest, Hungary
} 


\title{
Key Words
}

Intracranial aneurysm · Flow diverter $\cdot$ Neurological morbidity and mortality

\begin{abstract}
Background and Objective: Few prospective studies exist evaluating the safety and efficacy of the Pipeline Embolization Device (PED) in the treatment of intracranial aneurysms. The Aneurysm Study of Pipeline In an observational Registry (ASPIRe) study prospectively analyzed rates of complete aneurysm occlusion and neurologic adverse events following PED treatment of intracranial aneurysms. Materials and Methods: We performed a multicenter study prospectively evaluating patients with unruptured intracranial aneurysms treated with PED. Primary outcomes included (1) spontaneous rupture of the Pipeline-treated aneurysm; (2) spontaneous nonaneurysmal intracranial hemorrhage (ICH); (3) acute ischemic stroke; (4) parent artery stenosis, and (5) permanent cranial neuropathy. Secondary endpoints were (1) treatment success and (2) morbidity and mortality at the 6-month follow-up. Vascular imaging was evaluated at an independent core laboratory. Results: One hundred and ninety-one patients with 207 treated aneurysms were included in this registry. The mean aneurysm size was $14.5 \pm 6.9$ $\mathrm{mm}$, and the median imaging follow-up was 7.8 months. Twenty-four aneurysms (11.6\%) were small, 162 (78.3\%) were large and 21 (10.1\%) were giant. The median clinical follow-up time was 6.2 months. The neurological morbidity rate was $6.8 \%(13 / 191)$, and the neurological mortality rate was $1.6 \%(3 / 191)$. The combined neurological morbidity/mortality rate was $6.8 \%$ $(13 / 191)$. The most common adverse events were ischemic stroke $(4.7 \%, 9 / 191)$ and spontaneous ICH $(3.7 \%, 7 / 191)$. The complete occlusion rate at the last follow-up was $74.8 \%(77 / 103)$. Conclusions: Our prospective postmarket study confirms that PED treatment of aneurysms in a heterogeneous patient population is safe with low rates of neurological morbidity and mortality. Patients with angiographic follow-up had complete occlusion rates of $75 \%$ at 8 months.

(C) 2016 S. Karger AG, Base
\end{abstract}

\section{Introduction}

Treatment of intracranial aneurysms with the Pipeline Embolization Device (PED) is widely accepted as an excellent option for the treatment of intracranial aneurysms [1-4]. Flow diverter devices such as the PED were initially developed for the treatment of widenecked and large and giant aneurysms, aneurysms which are typically difficult to treat with endosaccular coiling. High rates of complete aneurysm occlusion have been reported in a number of studies, even in large and giant aneurysms [1-7].

Important questions remain regarding the safety and efficacy of flow diverter therapy. Even though numerous previous studies have reported overall rates of adverse events similar to those of other endovascular procedures, several studies documenting severe and 'unexpected' adverse events such as spontaneous intraparenchymal hemorrhage, spontaneous aneurysm rupture and postoperative strokes have raised questions about the safety of these devices [1, 3, 8-13].

The majority of existing literature on flow diversion therapy is comprised of single-center retrospective or prospective case series. Such literature has substantial biases, including selection and publication biases, which may affect apparent rates of angiographic occlusion and severe, unexpected adverse events. These biases may be diminished through the development of a prospective clinical registry. The purpose of the Aneurysm Study of Pipeline In an observational Registry (ASPIRe) study was to prospectively determine the rates of complete aneurysm occlusion and neurologic adverse events following the treatment of intracranial aneurysms with PED and validate results from previous clinical trials. 


\section{Materials and Methods}

\section{Study Design and Participants}

ASPIRe is a prospective, single-arm, multi-center postmarket registry of patients undergoing PED treatment of intracranial aneurysms. We prospectively evaluated all patients who were consented and treated with PED over a 3-year period in 28 centers in 7 countries experienced in PED use. Physicians who contributed data to this study were neurointerventionalists trained in endovascular techniques. As this was a multicenter registry, the following items varied across centers: selection of patients eligible for the treatment with the PED versus other treatment modalities, procedural details and periprocedural patient management. All centers used a common study protocol, which specified the data to be collected, study endpoints, events of interest and statistical analysis.

Patients were eligible for enrollment in the study if they: (1) consented to being included in the registry; (2) met the requirements for PED treatment per the instructions for use approved for the country in which they were treated, and (3) were willing and able to comply with follow-up visits. Patients were excluded if they (1) had an active bacterial infection; (2) had a contraindication to dual antiplatelet therapy or did not receive preoperative dual antiplatelet therapy; (3) had a preexisting stent in the parent artery at the target aneurysm location; (4) had a severe pre- or postaneurysmal narrowing, or (5) if the target aneurysm was acutely ruptured.

\section{Data Collection and Outcomes}

The baseline characteristics studied included medical history, demographic characteristics, presenting aneurysm location/size/type/rupture status, prior aneurysm treatment, concomitant medication use and presurgical imaging data. The operative characteristics studied included number and size of PEDs used, how PEDs were used (overlapping, multiple layers, etc.), side branch coverage, concomitant coiling, procedure duration and use of ancillary devices.

The primary study endpoint was comprised of the following: (1) spontaneous rupture of the Pipelinetreated aneurysm; (2) spontaneous nonaneurysmal intracranial hemorrhage (ICH) ipsilateral or contralateral to the treated aneurysm; (3) acute ischemic stroke; (4) symptomatic or asymptomatic parent artery stenosis, and (5) permanent cranial neuropathy. The secondary endpoints were (1) treatment success defined as complete occlusion of the Pipeline-treated aneurysm at the last follow-up and (2) morbidity and mortality at the 6-month follow-up. All vascular imaging was evaluated at an independent core laboratory for assessment of aneurysm occlusion and parent artery stenosis. Aneurysm occlusion was assessed using the scale of Roy and Raymond.

All adverse events were collected using a standard case report form. An adverse event was defined as any decline of the patient's baseline neurological status. Adverse events were defined as minor if the clinical sequelae of the complication resolved within 7 days and as major if the patient experienced a clinical deficit for $>7$ days. The relationship of the primary-endpoint adverse events and deaths to the procedure and device was established by an independent Clinical Events Committee.

Clinical and imaging follow-up time points were at the discretion of the operator. Clinical follow-up intervals were defined as follows: (1) baseline (before surgery); (2) surgery (at the time of surgery); (3) soon after surgery (up to 30 days after the procedure); (4) long after surgery (31-100 days after the procedure); (5) mid-term follow-up (101-250 days after the procedure), and (6) long-term follow-up (250 days or longer). Imaging follow-up intervals were defined as follows: 6 months $(-20 /+42$ days after the procedure $)$ and 1 year $( \pm 42$ days after the procedure).

\section{Loss to Follow-Up and Subject Withdrawal}

Subjects were considered lost to follow-up if they could not be reached after 3 attempts to contact the subjects at least 1 week apart. The final documented attempt was to be made with a registered letter. All enrolled subjects had the right to withdraw their consent. All data up to the time of withdrawal could be used for analysis. Any patients with a protocol deviation in data reporting, inclusion/exclusion criteria and informed consent were to be excluded from the analysis. No patients were excluded from our study due to protocol deviations as there were no inclusion/exclusion criteria violations and all informed consent deviations were minor and administrative in nature. 
Table 1. Patient characteristics

$$
\begin{array}{lc}
\text { Age, years } & \\
\text { Mean } \pm \text { SD } & 59.9 \pm 12.5(191) \\
\text { Median (min, max) } & 60.0(25.0,89.0) \\
\text { Gender } & 16.2(31 / 191) \\
\quad \text { Male } & 83.8(160 / 191) \\
\text { Female } & \\
\text { Race } & 86.7(157 / 181) \\
\text { White } & 7.2(13 / 181) \\
\text { Black or African American } & 1.7(3 / 181) \\
\text { Asian } & 0.6(1 / 181) \\
\text { American Indian or Alaska Native } & 0.6(1 / 181) \\
\text { Native Hawaiian or other Pacific Islander } & 3.3(6 / 181) \\
\text { Other } & \\
\text { Ethnicity } & 17.5(28 / 160) \\
\text { Hispanic or Latino } & \\
\text { Hypertension } & 53.9(103 / 191) \\
\text { Yes } & 93.2(96 / 103) \\
\quad \text { Controlled } & 41.9(80 / 191) \\
\quad \text { No } & 4.2(8 / 191) \\
\text { Unknown } & 43.7(80 / 183)
\end{array}
$$

Values are expressed as percentages with numbers in parentheses, unless otherwise indicated.

Statistical Analysis

All statistical analyses were performed using Statistical Analysis System (SAS) for Windows (version 9.2; SAS Institute Inc. Cary, N.C., USA). In general, data for all study subjects combined are presented. Data analysis is based on the subject level, except for aneurysm characteristics, which were based on the number of aneurysms.

Descriptive statistics are used to present the data and to summarize the results. Discrete variables are presented using frequency distributions and cross tabulations. Continuous variables are summarized by presenting the number of observations, mean, standard deviation, median, minimum and maximum values.

Statement of Ethics

Local institutional review boards or ethics committees approved the study and the use of the patients' data. Written informed consent was obtained from all study participants using a form approved by the local institutional review board or ethics committee.

\section{Results}

\section{Baseline Patient and Aneurysm Characteristics}

A total of 191 patients with 207 treated aneurysms were included in this registry. No patients were lost to clinical follow-up. The mean patient age was $59.9 \pm 12.5$ years. 160 patients $(83.8 \%)$ were female. Hypertension was present in $53.9 \%$ (103/191 patients), and $43.7 \%$ (80/183 patients) were current or previous smokers. The mean aneurysm size was $14.5 \pm 6.9 \mathrm{~mm}$ (range 0.9-41.0). Twenty-four aneurysms (11.6\%) were small, 162 (78.3\%) were large and $21(10.1 \%)$ were giant. The mean aneurysm neck size was $7.1 \pm 4.2 \mathrm{~mm} .81 .6 \%$ of the aneurysms (169/207) were saccular. In terms of aneurysm location, 95.2\% (197/207) were located in the anterior circulation, with 89.4\% (185/207) located on the internal carotid artery (ICA; 185/207). All treated aneurysms were unruptured. Median clinical follow-up time was 6.2 months (range 0.0-27.2). These data are summarized in tables 1 and 2. 
Table 2. Aneurysm characteristics

$\begin{array}{lc}\text { Aneurysm size, mm } & \\ \text { Mean } \pm \text { SD } & 14.5 \pm 6.9(207) \\ \text { Median (min, max) } & 12.0(0.9,41.0) \\ \text { Aneurysm neck, mm } & \\ \text { Mean } \pm \text { SD } & 7.1 \pm 4.2 \\ \quad \text { Median (min, max) } & 6.0(0.0,32.0) \\ \text { Aneurysm size } & 11.6(24 / 207) \\ \text { Small } & 78.3(162 / 207) \\ \text { Large } & 10.1(21 / 207) \\ \text { Giant } & \\ \text { Aneurysm type } & 81.6(169 / 207) \\ \text { Saccular } & 15.5(32 / 207) \\ \text { Fusiform } & 2.9(6 / 207) \\ \text { Dissecting } & \\ \text { Aneurysm side } & 54.1(112 / 207) \\ \text { Left } & 5.3(11 / 207) \\ \text { Midline } & 40.6(84 / 207) \\ \text { Right } & \\ \text { Aneurysm location } & 90.8(188 / 207) \\ \text { ICA } & 0.5(1 / 207) \\ \text { Anterior cerebral artery } & 1.4(3 / 207) \\ \text { Middle cerebral artery } & 0.5(1 / 207) \\ \text { Posterior cerebral artery } & 2.9(6 / 207) \\ \text { Basilar artery } & 1.4(3 / 207) \\ \text { Vertebral artery } & 2.4(5 / 207) \\ \text { Anterior communicating artery } & \\ \text { Aneurysm status } & 0.0(0 / 207) \\ \text { Ruptured } & 100.0(207 / 207) \\ \text { Unruptured } & \end{array}$

Values are expressed as percentages with numbers in parentheses, unless otherwise indicated.

\section{Procedure Characteristics}

Mean procedure time was $112.9 \pm 54.9 \mathrm{~min} .97 .9 \%$ of the patients $(187 / 191)$ were on anticoagulation/antiplatelet therapy. $66.5 \%$ of the patients $(127 / 191)$ were treated with the PED alone. In $18.8 \%$ of the cases (39/207), multiple PEDs were used. Side branches were covered by the PED in $50.7 \%$ of the cases $(104 / 205)$. Immediate post-treatment angiograms evaluated by the core laboratory demonstrated residual filling of the aneurysm in $86.9 \%$ of the cases $(166 / 191)$ and a neck remnant in $8.4 \%$ of the cases $(16 / 191)$. These data are summarized in table 3.

\section{Clinical and Imaging Outcomes}

Major adverse events occurred in $6.8 \%$ of the patients (13/191), and minor adverse events occurred in $4.7 \%$ of the patients $(9 / 191)$. Thirteen patients with major adverse events suffered neurological morbidity, 3 of whom suffered neurological mortality. Thus, the 6-month major neurological morbidity rate was 6.8\% (13/191), the neurological mortality rate was $1.6 \%(3 / 191)$ and the combined neurological morbidity/mortality rate was $6.8 \%$ (13/191). There were no additional cases of neurological morbidity and mortality beyond 6 months. In patients with saccular aneurysms (excluding dissecting and fusiform aneurysms), the neurological morbidity and mortality rate was $5.8 \%(9 / 156)$, and in patients with dissecting or fusiform aneurysms, the neurological morbidity and mortality rate was $10.8 \%$ $(4 / 37)$. 
Table 3. Procedure characteristics

\begin{tabular}{lc} 
Procedure time, min & $112.9 \pm 54.9(190)$ \\
Mean \pm SD & $107.0(0.0,369.0)$ \\
Median (min, max) & $97.9(187 / 191)$ \\
Anticoagulation/antiplatelet therapy & $61.3(117 / 191)$ \\
Platelet aggregation assay & $87.4(167 / 191)$ \\
Heparin during procedure & $16.2(27 / 167)$ \\
If yes, was heparin reversed? & $2.1(4 / 191)$ \\
Intraoperative imaging & $2.1(4 / 191)$ \\
Magnetic resonance angiography & $9.4(18 / 191)$ \\
Magnetic resonance imaging & $12.0(23 / 191)$ \\
Computed tomography & $98.4(188 / 191)$ \\
Computed tomography angiography & $6.3(12 / 191)$ \\
Angiogram & \\
Other & $1.0(2 / 191)$ \\
Level of occlusion (Core Laboratory reported) & $8.4(16 / 191)$ \\
100\% occlusion & $86.9(166 / 191)$ \\
Neck remnant & $3.7(7 / 191)$ \\
Residual filling & \\
Indeterminate or not available & \\
\hline Device characteristics & $66.5(127 / 191)$ \\
Type of treatment & $17.3(33 / 191)$ \\
PED alone & $10.5(20 / 191)$ \\
PED with coils & $5.8(11 / 191)$ \\
PED with balloons & \\
Other & $1.2 \pm 0.6(207)$ \\
Number of PEDs utilized & $1.0(0.0,5.0)$ \\
Mean \pm SD & $18.8(39 / 207)$ \\
Median (min, max) & $6.4(13 / 202)$ \\
Multiple PEDs utilized & \\
Multiple PEDs preplanned & $35.9(14 / 39)$ \\
Multiple PEDs, method of use & $51.3(20 / 39)$ \\
Additional length & $12.8(5 / 39)$ \\
Multiple layers & $94.2(194 / 206)$ \\
Stabilization & $50.7(104 / 205)$ \\
Entire neck covered by PEDs & $0.9 \pm 0.6(70)$ \\
Side branch covered by PEDs & $1.0(0.0,3.0)$ \\
Size of side branch from aneurysm sac, mm & \\
Mean \pm SD & \\
Median (min, max) & \\
& \\
&
\end{tabular}

Values are expressed as percentages with numbers in parentheses, unless otherwise indicated.

The most common adverse event was ischemic stroke, which occurred in $4.7 \%$ of the patients $(9 / 191)$ with stroke, resulting in major morbidity in only $1.6 \%$ of the patients $(3 / 191)$. The timing of ischemic stroke was surgical/early postoperative in 7 patients, late postoperative in 2 patients, and during mid-term follow-up in 1 patient. The time range for acute ischemic stroke was 0-238 days after the procedure. One patient suffered two ischemic strokes. The acute ischemic stroke rate was $0 \%(0 / 10)$ in subjects with aneurysms in the posterior circulation and $1.7 \%(3 / 181)$ in subjects with aneurysms in the anterior circulation $(p=1.00)$.

Spontaneous ICH occurred in $3.7 \%$ of the patients $(7 / 191)$. There were a total of nine ICHs in these 7 patients. ICH resulted in major morbidity in all 7 patients. The timing of ICH ranged from 0-103 days and was in the surgical/early postoperative period for 7 events in 6 patients, in the late postoperative period for 1 event in 1 patient and during mid-term follow- 
Table 4. Adverse events

\begin{tabular}{llc}
\hline Adverse events of interest & Major & Minor \\
\hline Ischemic stroke & $1.6(3 / 191)$ & $3.1(6 / 191)$ \\
ICH & $3.7(7 / 191)$ & $0.0(0 / 191)$ \\
$\quad$ Ipsilateral ICH & $3.1(6 / 191)$ & $0.0(0 / 191)$ \\
$\quad$ Contralateral ICH & $0.5(1 / 191)$ & $0.0(0 / 191)$ \\
Asymptomatic parent artery stenosis & $0.0(0 / 191)$ & $1.6(3 / 191)$ \\
Symptomatic parent artery stenosis & $0.0(0 / 191)$ & $0.0(0 / 191)$ \\
Spontaneous rupture & $1.6(3 / 191)$ & $0.0(0 / 191)$ \\
Permanent cranial neuropathy & $0.0(0 / 191)$ & $0.0(0 / 191)$ \\
\hline Total events, $\mathrm{n}$ & 15 & 9 \\
\hline Total subjects & $6.8(13 / 191)$ & $4.7(9 / 191)$ \\
\hline
\end{tabular}

Values are presented as percentages with numbers in parentheses. Percentages are reported on a per-patient basis. There were a total of 9 ICHs in 7 subjects: 1 subject experienced two major ICHs on different days; 1 subject had two ICHs, one was major and one could not be categorized as 'major' or 'minor'.

Table 5. Timing of major adverse events

\begin{tabular}{llrrr}
\hline Major adverse events of interest & $<3$ days & $3-30$ days & $>30$ days & Total \\
\hline Ischemic stroke & $1.0(2 / 191)$ & $0.5(1 / 191)$ & $0(0 / 191)$ & $1.6(3 / 191)$ \\
ICH & $2.6(5 / 191)$ & $0.5(1 / 191)$ & $0.5(1 / 191)$ & $3.7(7 / 191)$ \\
Asymptomatic parent artery stenosis & $0.0(0 / 191)$ & $0.0(0 / 191)$ & $0.0(0 / 191)$ & $0.0(0 / 191)$ \\
Symptomatic parent artery stenosis & $0.0(0 / 191)$ & $0.0(0 / 191)$ & $0.0(0 / 191)$ & $0.0(0 / 191)$ \\
Spontaneous rupture & $0.5(1 / 191)$ & $1.0(2 / 191)$ & $0(0 / 191)$ & $1.6(3 / 191)$ \\
Permanent cranial neuropathy & $0.0(0 / 191)$ & $0.0(0 / 191)$ & $0.0(0 / 191)$ & $0.0(0 / 191)$ \\
Neurologic morbidity & $4.2(8 / 191)$ & $2.1(4 / 191)$ & $0.5(1 / 191)$ & $6.8(13 / 191)$ \\
Neurologic mortality & $0(0 / 191)$ & $1.0(2 / 191)$ & $0.5(1 / 191)$ & $1.6(3 / 191)$ \\
Neurologic morbidity and mortality & $4.2(8 / 191)$ & $2.1(4 / 191)$ & $0.5(1 / 191)$ & $6.8(13 / 191)$ \\
\hline
\end{tabular}

Values are expressed as percentages with numbers in parentheses.

up for 1 event in the same patient. Among the 7 subjects with ICH, 6 had hemorrhage occurring ipsilateral to the PED, and 1 had a contralateral hemorrhage. Spontaneous rupture occurred in $1.6 \%$ of the patients $(3 / 191)$, resulting in major morbidity in all cases. All cases of spontaneous rupture occurred within 4 days of the procedure. One of these patients was concomitantly coiled, and none were previously coiled.

There were 3 cases of minor asymptomatic parent artery stenosis (1.6\%) and no cases of permanent cranial neuropathy $(0.0 \%)$. Twenty-one of the 25 primary adverse events $(84 \%)$ were determined to be device- or procedure-related by the Clinical Events Committee. These data are summarized in tables 4 and 5.

There were no statistically significant differences in the rates of acute ischemic stroke, ICH or spontaneous aneurysm rupture between subjects who underwent different technical aspects of the procedure including: use of single or multiple PEDs, whether side branches were covered, whether the entire neck of the aneurysm was covered and whether the PED landed in the intended location. There were significant differences in the rates of acute ischemic stroke, spontaneous aneurysm rupture and neurological death based on aneurysm size. Subjects with giant aneurysms ( $\geq 25 \mathrm{~mm}$ ) had significantly higher rates of acute ischemic 
stroke $(p=0.03)$, spontaneous aneurysm rupture $(p=0.03)$ and neurological death $(p=0.03)$ compared to subjects with small and large aneurysms. There was no significant difference in the rate of ICH between subjects with giant aneurysms and those with small and large aneurysms. Further, there was no significant difference in the rates of acute ischemic stroke, ICH, spontaneous aneurysm rupture or neurological death between subjects with small aneurysms and those with large aneurysms.

Imaging follow-up of at least 6 months was obtained in 103 subjects (54\%). The complete occlusion rate at the last follow-up, which occurred at a median time of 7.8 months (mean 9.7 $\pm 4.2)$ after the procedure, was $74.8 \%(77 / 103)$. Among patients who had their last imaging follow-up at 6 months, the complete occlusion rate was 78.6\% (33/42), and among patients who had their last imaging follow-up at 1 year, the complete occlusion rate was $79.0 \%$ $(15 / 19)$. Eleven $(5.8 \%)$ patients required retreatment.

\section{Discussion}

Our study demonstrated that the treatment of unruptured intracranial aneurysms with the PED in a broad postmarket setting is safe. The complete occlusion rates were nearly $75 \%$ for patients with angiographic follow-up, with a median of 7.8 months of follow-up. Major adverse events occurred in $<7 \%$ of the patients with $\mathrm{ICH}$, and the stroke rates were $<4$ and $2 \%$. Spontaneous rupture was rare, occurring in $1.6 \%$ of the patients. Approximately $80 \%$ of the major adverse events, particularly hemorrhages and spontaneous ruptures, occurred in the early postoperative period. These findings are important as they provide further data regarding the incidence of severe adverse events associated with flow diversion in a realworld setting with a broad range of aneurysm sizes and locations.

Our safety findings corroborate the findings of other large multicenter studies and metaanalyses including the International Retrospective Study of Pipeline Embolization Device registry (IntrePED) and the Pipeline for Uncoilable or Failed Aneurysms study (PUFS) [13, 14]. Long-term neurological morbidity and mortality rates were $8.4 \%$ in the IntrePED and $5.6 \%$ in the PUFS compared to $6.8 \%$ in our study. When patients with ruptured, dissecting or fusiform aneurysms were excluded in IntrePED, the overall neurological morbidity and mortality rate in IntrePED dropped down to 5.7\%, similar to our study [13]. Two large metaanalyses of flow diverter treatment demonstrated morbidity rates of $5.0-7.3 \%$ and mortality rates of $2.8-4.0 \%[1,3]$.

The rate of spontaneous rupture was slightly higher in our study when compared to IntrePED (1.6 vs. $0.6 \%$ ) but was substantially lower than the $3 \%$ rate reported in the metaanalysis performed by Brinjikji et al. [3]. Similar to our study, PUFS reported a spontaneous aneurysm rupture rate of $1.9 \%$ [14]. The rate of ICH in ASPIRe was slightly higher than the rate in IntrePED (3.7 vs. $2.5 \%$ ) but lower than that reported in PUFS (4.7\%). Similar to both PUFS and IntrePED, a majority of spontaneous ICHs occurred in the early postoperative period.

Nearly $75 \%$ of the aneurysms in our study were large aneurysms of the ICA. Prior studies have demonstrated high angiographic cure rates when treating large ICA aneurysms with flow diverters such as the PED. Complete occlusion rates in studies reporting 6-to-12-month follow-ups typically range from 70 to $93 \%$ [14-19]. One large meta-analysis of nearly 1,500 patients with 1,700 aneurysms treated with flow diverters found complete occlusion rates of $76 \%$ at the last follow-up. One series of 38 aneurysms with medium-term follow-up reported complete occlusion of all 27 ICA aneurysms by 18 months with progressively increased occlusion between 3-18 months of follow-up [20]. In their study of 251 large and giant aneurysms undergoing PED treatment, Saatci et al. [21] reported a 91.2\% complete occlusion rate at 6 months and a $94.6 \%$ complete occlusion rate at 1-2 years after the treatment. 
Other endovascular treatments available for the treatment of wide-necked large and giant ICA aneurysms include stent-assisted endosaccular coiling as well as endovascular parent artery occlusion (PAO). In a series of 56 consecutive patients receiving PAO for the treatment of large/giant aneurysms of the carotid siphon, Labeyrie et al. [22] demonstrated an aneurysm retraction rate of $91 \%$ and a procedure-related permanent morbidity rate of $5 \%$. Twenty-six percent of the patients in the Labeyrie series [22] suffered ischemic events, with symptoms resolving in most patients. Other series report ischemic stroke rates of $5-15 \%$ for PAO of ICA aneurysms with permanent occlusion rates of 90-100\% [23-25]. While the rates of permanent aneurysm occlusion and procedure-related morbidity rates in the above series are similar to those of large and giant aneurysms in PUFS, it is important to emphasize that PAO techniques can only be used in patients who can tolerate occlusion of the carotid artery. By preserving parent artery flow, flow diverters such as the PED can be used in the treatment of patients in both the presence and absence of collateral flow [22].

Like flow diverter treatment, endosaccular and stent-assisted coiling allow for preservation of the parent artery flow. However, one major disadvantage of endosaccular coiling is the high rates of recanalization, especially when stent assistance is not used. In our study, there was a low rate of retreatment. In a meta-analysis of treatment of cavernous carotid artery aneurysms with endosaccular coiling and PAO, Turfe et al. [26] found long-term aneurysm complete occlusion rates of just $45.0 \%$ with non-stent-assisted endosaccular coiling and retreatment rates of $20.0 \%$. Stent-assisted coiling resulted in a $56.0 \%$ complete occlusion rate and a retreatment rate of $22 \%$ [26]. Procedure-related morbidity and mortality were $<5 \%$ for patients undergoing coiling. A meta-analysis of patients undergoing stentassisted coiling versus coiling only found that stent-assisted coiling recurrence rates were $16.2 \%$ compared to $34.4 \%$ in non-stent-assisted coiling. Complication rates were not negligible with mortality rates ranging from 3-9\% and permanent morbidity rates ranging from 4-6\% [27]. In a series of over 150 large and giant ICA aneurysms treated with coil embolization, Chalouhi et al. [28] noted a $12 \%$ complication rate with recurrence and retreatment rates $>30 \%$. D'Urso et al. [29] demonstrated a complete occlusion rate of just $62 \%$ for ICA aneurysms treated with coil embolization and a 5\% complication rate. When compared to the results of the PUFS trial, it is clear that despite their ability to preserve parent artery flow and low complication rates, coiling techniques with and without stent assistance have lower rates of angiographic occlusion and higher rates of recurrence and retreatment when compared to PED placement alone [14].

\section{Limitations}

Our study has limitations. This study was a prospective multicenter registry in which sites followed their standard of practice for treating aneurysms with PED and there was a wide range of treatment regimens (i.e. platelet testing and antiplatelet therapy) between centers. However, all study adverse events collected were prespecified and evaluated by an independent Clinical Events Committee to maintain consistency. As study eligibility was limited to the approved indication in the country of treatment, this registry did not capture use of the PED in patients treated off-label. Another potential limitation of this study is the fact that the indications for treatment varied from country to country, which can result in a degree of selection bias. Although there was a core laboratory for standardization of image reads, there was no protocol regarding the minimum angiographic follow-up as this was left to the discretion of the operator. Thus, there is substantial heterogeneity in the timing of the last imaging follow-ups with just over $50 \%$ of the patients having angiographic imaging follow-up at 6 months or later, thus limiting our evaluation of occlusion outcomes. However, unlike many other previously published studies, all patients included in our study had clinical follow-up, and no patients were lost to follow-up. 


\section{Conclusions}

Our postmarket study of PED treatment of a broad range of intracranial aneurysm sizes and locations confirms that the treatment of intracranial aneurysms with the PED is both safe and effective with complete occlusion rates of approximately $75 \%$ at 7.8 months and low rates of neurological morbidity and mortality. The neurological morbidity and mortality rate drops further when patients with difficult-to-treat aneurysms (dissecting or fusiform) are excluded. These findings should be considered when determining the best therapeutic option for intracranial aneurysms.

\section{Acknowledgments}

This observational registry was funded and supported by Covidien, with scientific oversight of the study steering committee members. This study was funded by Medtronic, and D.F.K. receives research funding from Medtronic.

\section{Disclosure Statement}

The authors declare that there are no conflicts of interest to disclose.

\section{References}

1 Arrese I, Sarabia R, Pintado R, et al: Flow-diverter devices for intracranial aneurysms: systematic review and meta-analysis. Neurosurgery 2013;73:193-200.

2 Briganti F, Napoli M, Tortora F, et al: Italian multicenter experience with flow-diverter devices for intracranial unruptured aneurysm treatment with periprocedural complications - a retrospective data analysis. Neuroradiology 2012;54:1145-1152.

3 Brinjikji W, Murad MH, Lanzino G, et al: Endovascular treatment of intracranial aneurysms with flow diverters: a meta-analysis. Stroke 2013;44:442-447.

4 Yu SC, Kwok CK, Cheng PW, et al: Intracranial aneurysms: midterm outcome of pipeline embolization device - a prospective study in 143 patients with 178 aneurysms. Radiology 2012;265:893-901.

5 Chan TT, Chan KY, Pang PK, et al: Pipeline embolisation device for wide-necked internal carotid artery aneurysms in a hospital in Hong Kong: preliminary experience. Hong Kong Med J 2011;17:398-404.

6 Cirillo L, Dall'olio M, Princiotta C, et al: The use of flow-diverting stents in the treatment of giant cerebral aneurysms: preliminary results. Neuroradiol J 2010;23:220-224.

7 Siddiqui AH, Kan P, Abla AA, et al: Complications after treatment with pipeline embolization for giant distal intracranial aneurysms with or without coil embolization. Neurosurgery 2012;71:E509-E513; discussion E13.

8 Cruz JP, Chow M, O’Kelly C, et al: Delayed ipsilateral parenchymal hemorrhage following flow diversion for the treatment of anterior circulation aneurysms. AJNR Am J Neuroradiol 2012;33:603-608.

9 Pierot L: Flow diverter stents in the treatment of intracranial aneurysms: where are we? J Neuroradiol 2011; 38:40-46.

10 Darsaut TE, Rayner-Hartley E, Makoyeva A, et al: Aneurysm rupture after endovascular flow diversion: the possible role of persistent flows through the transition zone associated with device deformation. Interv Neuroradiol 2013;19:180-185.

11 Kuzmik GA, Williamson T, Ediriwickrema A, et al: Flow diverters and a tale of two aneurysms. J Neurointerv Surg 2013;5:e23.

12 Turowski B, Macht S, Kulcsar Z, et al: Early fatal hemorrhage after endovascular cerebral aneurysm treatment with a flow diverter (SILK-Stent): do we need to rethink our concepts? Neuroradiology 2011;53:37-41.

13 Kallmes DF, Hanel R, Lopes D, et al: International retrospective study of the pipeline embolization device: a multicenter aneurysm treatment study. AJNR Am J Neuroradiol 2015;36:108-115.

14 Becske T, Kallmes DF, Saatci I, et al: Pipeline for uncoilable or failed aneurysms: results from a multicenter clinical trial. Radiology 2013;267:858-868.

15 Puffer RC, Piano M, Lanzino G, et al: Treatment of cavernous sinus aneurysms with flow diversion: results in 44 patients. AJNR Am J Neuroradiol 2014;35:948-951. 
16 Moon K, Albuquerque FC, Ducruet AF, et al: Resolution of cranial neuropathies following treatment of intracranial aneurysms with the Pipeline Embolization Device. J Neurosurg 2014;121:1085-1092.

17 Szikora I, Berentei Z, Kulcsar Z, et al: Treatment of intracranial aneurysms by functional reconstruction of the parent artery: the Budapest experience with the pipeline embolization device. AJNR Am J Neuroradiol 2010; 31:1139-1147.

18 Lylyk P, Miranda C, Ceratto R, et al: Curative endovascular reconstruction of cerebral aneurysms with the pipeline embolization device: the Buenos Aires experience. Neurosurgery 2009;64:632-642; discussion 42-43; quiz N6.

19 Nelson PK, Lylyk P, Szikora I, et al: The pipeline embolization device for the intracranial treatment of aneurysms trial. AJNR Am J Neuroradiol 2011;32:34-40.

20 Briganti F, Napoli M, Leone G, et al: Treatment of intracranial aneurysms by flow diverter devices: long-term results from a single center. Eur J Radiol 2014;83:1683-1690.

21 Saatci I, Yavuz K, Ozer C, et al: Treatment of intracranial aneurysms using the pipeline flow-diverter embolization device: a single-center experience with long-term follow-up results. AJNR Am J Neuroradiol 2012;33: $1436-1446$

22 Labeyrie MA, Lenck S, Bresson D, et al: Parent artery occlusion in large, giant, or fusiform aneurysms of the carotid siphon: clinical and imaging results. AJNR Am J Neuroradiol 2015;36:140-145.

23 Clarencon F, Bonneville F, Boch AL, et al: Parent artery occlusion is not obsolete in giant aneurysms of the ICA. Experience with very-long-term follow-up. Neuroradiology 2011;53:973-982.

24 van der Schaaf IC, Brilstra EH, et al: Endovascular treatment of aneurysms in the cavernous sinus: a systematic review on balloon occlusion of the parent vessel and embolization with coils. Stroke 2002;33:313-318.

25 van Rooij WJ: Endovascular treatment of cavernous sinus aneurysms. AJNR Am J Neuroradiol 2012;33:323326.

26 Turfe ZA, Brinjikji W, Murad MH, et al: Endovascular coiling versus parent artery occlusion for treatment of cavernous carotid aneurysms: a meta-analysis. J Neurointerv Surg 2015;7:250-255.

27 Hong Y, Wang YJ, Deng Z, et al: Stent-assisted coiling versus coiling in treatment of intracranial aneurysm: a systematic review and meta-analysis. PLoS One 2014;9:e82311.

28 Chalouhi N, Tjoumakaris S, Gonzalez LF, et al: Coiling of large and giant aneurysms: complications and longterm results of 334 cases. AJNR Am J Neuroradiol 2014;35:546-552.

29 D'Urso PI, Karadeli HH, Kallmes DF, et al: Coiling for paraclinoid aneurysms: time to make way for flow diverters? AJNR Am J Neuroradiol 2012;33:1470-1474. 